\title{
Low-Reflection Cross Section and High-Isolation 2x2 Broadband Antenna Array for the MIMO Measurement System
}

\author{
Wen-Yu Lee, a, Hsin-Piao Lin ${ }^{1}$ and Ding-Bing Lin ${ }^{1}$ \\ ${ }^{1}$ Department of Electronic Engineering, Taipei University of Technology, Taipei, Taiwan
}

\begin{abstract}
The author of this paper explored Vivaldi [1] [2] line theory and technology and used it as a basis to propose a Vivaldi antenna array to replace a single Vivaldi antenna. This was to achieve a dual-polarized antenna with high directivity and high isolation in the MIMO anechoic chamber, and one that is minimally affected by the environment. The operating frequency of this antenna array covers a frequency range of $0.7-6.0 \mathrm{GHz}$ and is composed of four Vivaldi units with relatively high isolation between them to reduce measurement errors caused by coupling. In each unit, two identical Vivaldi antennas are connected in parallel to form the same polarization unit, and a microstrip power divider was used and the impedance matching of circular holes was performed to design this connected antenna with an ultra-wide operating frequency and the same polarization. The authors then interconnected two polarization units orthogonally at 90 degree cross to form the antenna described in this study, which has high directivity, high isolation, an ultra-wide frequency and dual polarization. During the design process, an FR4 printed circuit board (PCB) was used to effectively reduce the cross-sectional area of the antenna and reduce reflection and interference on the basis of ensuring an ultra-wide operating frequency. Additionally, the two orthogonal units of each polarized antenna unit had to work separately, and the electric field data collected from different polarization directions were sequentially transmitted to the receiver for postprocessing to satisfy the measurement requirements of the MIMO OTA anechoic chamber. In this study, SEMCAD electromagnetic simulation software was used to adjust and complete the analysis of antenna characteristics and obtain a favorable operating frequency and voltage standing wave ratio, as well as excellent isolation and radiation characteristics.
\end{abstract}

\section{Introduction}

The requirements of modern mobile communication technology for Multiple Input Multiple Output (MIMO) over the air (OTA) measurement methods and test precision are becoming more stringent. Mobile communication system MIMO OTA measurements are performed in indoor environments. Because the multichannel transmission pathways of reflection, diffraction, and refraction in the actual environment must be simulated to overcome the limitations of site space and distance in far-field measurements, multiple antennas must be set up in an anechoic chamber to transmit multiple sets of signals. The antenna array technology in an anechoic chamber was first proposed in the 1980s. In 2000, the French firm Satimo announced an anechoic chamber featuring antenna arrays at $0.7-6.0 \mathrm{GHz}$ (Fig. 1), leading to another revolution in antenna measurement technology. This technology arranges multiple antennas into an array in a three-dimensional space; replaces conventional single-probe, mechanical mobile measurements; increases the test rate; and prevents errors caused by inaccurate mechanical scanning.

Most conventional anechoic chambers employ a metal, open-boundary, broadband horn antenna. Due to its large volume and larger transmission cross section, the antenna can easily cause multipath reflection interference in electromagnetic wave propagation in the anechoic chamber, causing errors in the measurement results. Therefore, the present study designed a set of antennas with high directivity, high isolation, an ultra-wide band, and dual polarization, as well as relatively small transmission cross sections (Figs 2 and 3). During measurements in the anechoic chamber, this set of antennas effectively reduced multipath reflection in signals and mutual interference. Its structure consists of two identical Vivaldi antennas connected in parallel to form the same polarization unit. A microstrip power divider and circular hole impedance matching method were used to design this parallel antenna with an ultrawide working band and the same polarization. The authors of this paper designed a square metal reflector on the bottom of the antenna (Fig. 2) to increase its directivity and effectively reduce the multipath reflection of electromagnetic waves. Furthermore, the microstrip power divider line was used as the coupling and feed point of the signal source.

In 1979, Gibson [3] proposed a broadband, exponentially tapered microstrip Vivaldi slot antenna. Its opening curvature is an exponential taper, its slot has an

\footnotetext{
a Corresponding author: toyoant@yahoo.com.tw
} 
exponentially tapered structure, and it is also characterized by a wide band, high gain, and symmetrical beams [4]. The antenna in this paper was fabricated using a double-sided copper foil FR4 fiberglass printed circuit board (PCB). Because a PCB microstrip antenna is light and has a simple structure, it is widely used in the field of microwave transmission measurement systems. The microstrip line and signal distributor for signal feeding are printed on the front side of the PCB, whereas its exponential V-shaped open antenna body is printed on the other side of the PCB. Such a configuration design serves as an impedance transformation network for the signal feeding distributor and radiance propagation in free space, as well as the coupling feed of signals. The gap size of the narrower end of the exponential slot on the radiator of the antenna can determine the cutoff frequency (highest frequency), and the gap size of the wider end of the antenna body can determine the initial frequency (lowest frequency). Langley et al. [5] developed a stripline-fed Vivaldi antenna with a doublesided symmetrical structure. Additionally, Linardou et al. [6] proposed a coplanar waveguide-fed Vivaldi antenna. Based on the aforementioned publications, scholars have achieved several results in research work regarding Vivaldi antennas.

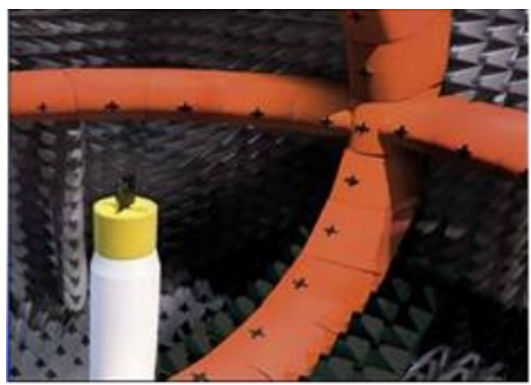

Figure 1. MIMO OTA EM wave anechoic chamber installed with 16 sets of antennas.

\section{Principle and Structure}

\subsection{Fundamental principle}

A Vivaldi antenna is a nonperiodic and tapered wave antenna. It uses its exponential slot to radiate outward or receive electromagnetic waves inward. For a certain operating frequency, only $\mathrm{V}$-shaped slots and areas with similar widths and wavelengths form the external space of the resonance term to generate radiation. When the operating frequency changes, its corresponding radiation area also changes. A Vivaldi antenna is a terminal radiating antenna type, the surface waves of which are transmitted to the open slot area, and its characteristics of radiation is different from microstrip patch and dipole antennas. Because the phase velocity of a surface wave is generally lower than the speed of light, the exponentially tapered slot antenna has a slow-wave structure. Compared with other antennas featuring constant surface wave phase velocity of propagation paths, this antenna has a more favorable rate of phase velocity, which enables the antenna to obtain stronger directivity and higher gain. The most unique advantage of the Vivaldi antenna, however, is its wider broadband. Because its radiation end has the tapered opening of an exponential slot, the propagation phase velocity changes progressively, thereby reducing its directivity and also causing the field strength of the minor lobe to decrease. The two main polarization planes parallel (E-Plane) and vertical (H-Plane) to the PCB antenna structure have a favorable symmetrical radiation pattern, and its radiation polarization is linear. When using a PCB with a higher dielectric constant, the size of the polarization planes can be reduced. If using a PCB with a different dielectric constant, the thickness of the polarization planes and changes in the dimensions and shape of the radiation opening can change the value of the halfvalue angle of radiation. This paper is based on a linear single-polarized array antenna with two identical Vivaldi antennas connected in parallel in a one-dimensional space (Fig. 3).

The Vivaldi antenna can be used as a single antenna by itself or as an array antenna (Fig. 6), or also as a single- or dual-polarized array antenna (Fig. 2). According to the principles of the microstrip transmission line and slot antenna, an analytical simulation and modifications were performed based on the Vivaldi antenna. The curvature of the exponentially changing slots and size of the circular holes were adjusted as an impedance matching method to enhance the performance of the Vivaldi antenna. The antenna was then modeled and simulated using the electromagnetic simulation software package SEMCAD.

\subsection{Structural design analysis}

The structural design of the antenna in this study can be divided into four layers, which are described as follows: the first layer is an FR4 fiberglass substrate plate; the second layer is an exponentially tapered slot antenna; the third is an FR4 fiberglass substrate plate; and the fourth is microstrip feeding circuit consisting of a power divider and copper foil microstrip line for signal feeding (Fig. 4). The antenna body engraves an exponentially tapered slot structure on the copper foil layer of the second layer. Its tapered slot curvature can be regarded as being composed of three parts: the first part is a circular hole for the adjustment of impedance matching of the antenna. The parallel slot of the second part serves as a signal feed coupling device that affects the insertion loss of the feed signal. The fan-shaped design of the third part is primarily the transmission line and antenna coupling (Fig. $5)$. 


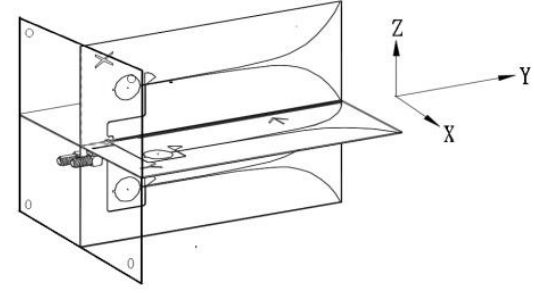

Figure 2. Dual-port dual-polarized antenna array with a metal reflector.
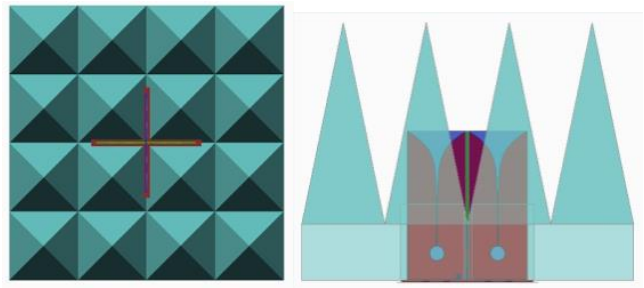

Figure 3. Antenna with smaller cross-section area along the propagation direction proposed in this study.

The determinants of the operating bandwidth of the antenna include the following: (1) exponentially tapered curvature of the radiator of the antenna; (2) fan-shaped coupling design; (3) microstrip line design; (4) circular hole impedance; and (5) PCB dielectric constant. The inclusion of an impedance matching design can effectively extend the bandwidth of the antenna and enhance its reflection loss and impedance matching characteristics.

The curvature relationship of the exponentially tapered V-shaped slots affects the characteristics of the antenna's operating broadband. The curvature shapes of the key design parameters were determined using the following equations:

$$
\begin{aligned}
& y=c_{1} e^{R x}+c_{2} \\
& c_{1}=\frac{y_{2}-y_{1}}{e^{R x_{2}}-e^{R x_{1}}} \\
& c_{2}=\frac{y_{1} e^{R x_{2}}-y_{2} e^{R x_{1}}}{e^{R x_{2}}-e^{R x_{1}}}
\end{aligned}
$$

Because the medium between the slots was FR-4 material, its dielectric constant was 4.2, and the relationship between its operating frequency $f H$ and wavelength and the slot width $W a$ is presented as the follow equation:

$$
W a / 4=\frac{c}{f_{H} \sqrt{\varepsilon_{e}}}
$$

The exponentially tapered slots of the antenna in Fig. 6 have two key parameters, P1 (x,z) and P2 (x,z), which are the starting and end points of the exponentially tapered curvature. The opening rate of exponential changes of the slots is $\mathrm{R}$ and the diameter of the circular cavity is Dr.

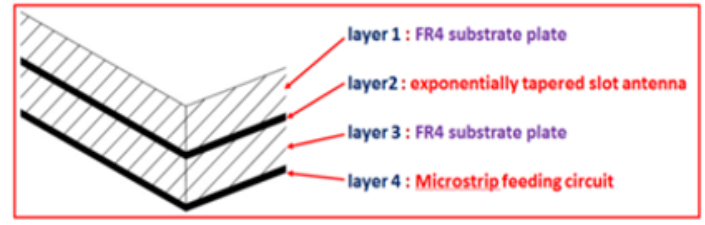

Figure 4. Side view of the actual structure and size of the PCB.

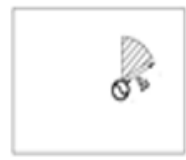

(1)

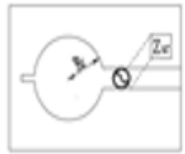

(2)

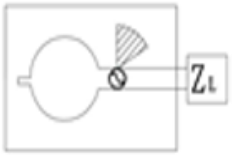

(3)
Figure 5. Structure of circular sector coupling and feeding circuits.

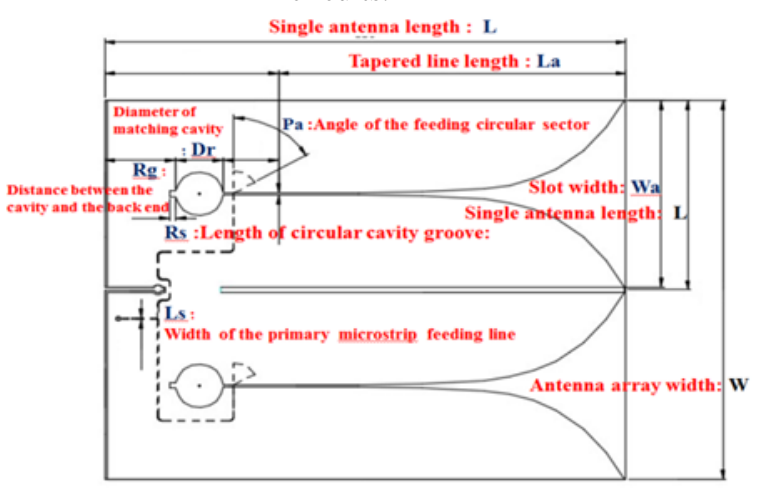

Figure 6. Antenna PCB cross-section diagram.

\section{Experiment and Modifications}

The PCB used by the antenna in this study had a dielectric constant of $\varepsilon r=4.2$, was made of FR4 fiberglass material with a thickness of $2.0 \mathrm{~mm}$, and had a microstrip line thickness of $0.5 \mathrm{oz}$. The bottommost surface in Fig. 4 is the copper foil layer conductor, and the center black line is the power divider and microstrip feed line of the reverse side. The microstrip feed line crosses the exponentially tapered slot line horizontally at 90 degrees, and connects to the coaxial cable through the L-shaped SAMJ connector soldered on the PCB, thereby feeding signals. In the present study, although the structure of the antenna is simple, the design and adjustment of its power divider and feeding circuit are relatively difficult. Differences in the design of this feeding circuit affect the operating bandwidth and insertion loss. The design and adjustment of this antenna was completed using SPEAG's high-frequency simulation software SEMCAD. After several dimension changes and computations, a set of structural dimensions that satisfied the requirements of this study were obtained, which are detailed as follows:

Table 1. Antenna structure dimensions. 


\begin{tabular}{|l|l|}
\hline Single antenna length: & $\mathrm{L}=180 . \mathrm{mm}$ \\
\hline Antenna array width: & $\mathrm{W}=140 . \mathrm{mm}$ \\
\hline Slot width: & $\mathrm{Wa}=70 . \mathrm{mm}$ \\
\hline Tapered line length: & $\mathrm{La}=120 . \mathrm{mm}$ \\
\hline Diameter of matching cavity: & $\mathrm{Dr}=16.5 \mathrm{~mm}$ \\
\hline Length of circular cavity groove: & $\mathrm{Rs}=2 . \mathrm{mm}$ \\
\hline Distance between the cavity and the back end: & $\mathrm{Rg}=23 . \mathrm{mm}$ \\
\hline Angle of the feeding circular sector: & $\mathrm{Pa}=55^{\circ}$ \\
\hline Width of the primary microstrip feeding line: & $\mathrm{Ls}=0.75 \mathrm{~mm}$ \\
\hline
\end{tabular}

The aforementioned parameters were used for simulation to obtain the full-bandwidth voltage standing wave ratio (VSWR) curvature and favorable values (Fig. 7). The simulation results indicated that within the operating bandwidth of $0.7-6.0 \mathrm{GHz}$, the VSWR was $\leq$ 2.5. The obtained full-bandwidth isolation values of the antenna were nearly all less than $-35 \mathrm{~dB}$ (Fig. 8). Excellent performance of $-40 \mathrm{~dB}$ was achieved at frequency bands above $2.0 \mathrm{GHz}$. The isolation at horizontally and vertically polarized poles interacted with the isolation at extremely low frequency. The researchers then changed the local size of the antenna to improve its characteristics. Because antenna dimensions such as L, W, $\mathrm{W}$ a, $\mathrm{L}$ a, R, and $\mathrm{Pa}$ affect the antenna characteristics, the effect of error on antenna characteristics was considered. The researchers only calculated the effects of the following on the VSWR of the antenna: microstrip feed line size, extension length of the microstrip feed line, and changes in the diameter $\mathrm{R}$ of the matching circular hole \pm $0.1 \mathrm{~mm}$. The results indicated that within a bandwidth range of $0.7-6.0 \mathrm{GHz}$, changes in the return loss of the antenna were minimized, and only slight changes were observed in the frequency point shift before and after (Figs 9-1, 9-2, and 9-3).

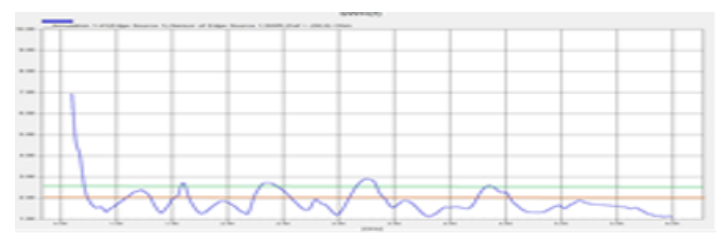

Figure 7. VSWR of final outcome after parameter optimization at $0.7-6.0 \mathrm{GHz}$

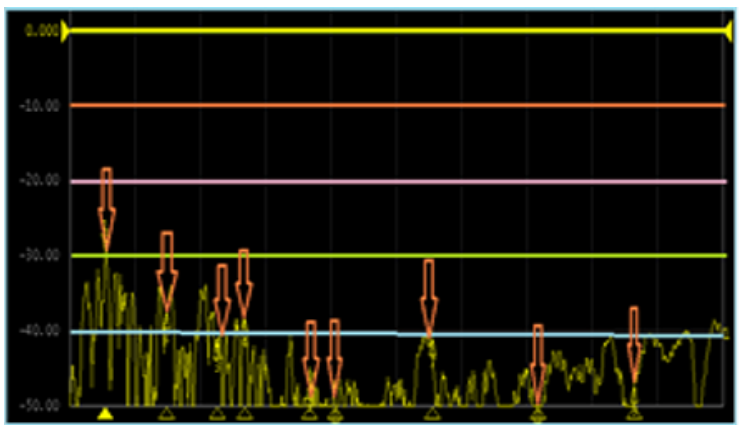

Figure 8. Isolation of $\mathrm{V} / \mathrm{H}$ ports at $0.7-6.0 \mathrm{GHz}$.

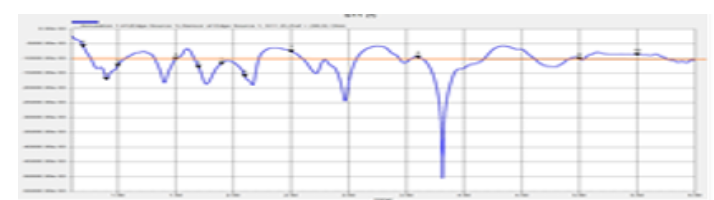

Figure 9-1. Return loss without modification.

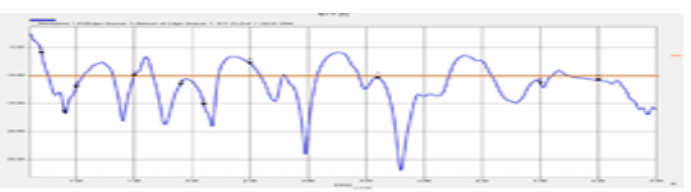

Figure 9-2. Return loss after the first modification.

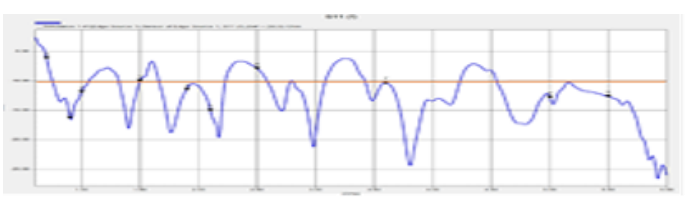

Figure 9-3. Return loss after the second modification.

\section{Data Analysis}

According to the aforementioned results, the researchers modified several sizes of microstrip antennas. The parameters and size affect the VSWR results of the actual antenna. Figs 9-1 to 9-3 show that increases in the size of the circular hole structure enhance the performance of the high-frequency end. Additionally, the return loss of all frequency points was less than $-8 \mathrm{~dB}$, which essentially satisfies the experimental requirements. In this paper, SEMCAD was employed to refer to the frequency bands used in modern mobile communication to select four frequency points representing low, medium, and high frequencies for the antenna in this study: $0.9,1.9,3.6$, and $5.5 \mathrm{GHz}$. After coating the electromagnetic wave absorber, favorable results were obtained through simulation calculations and modifications of the antenna set (as shown in Figs 10-1 to 13-2).

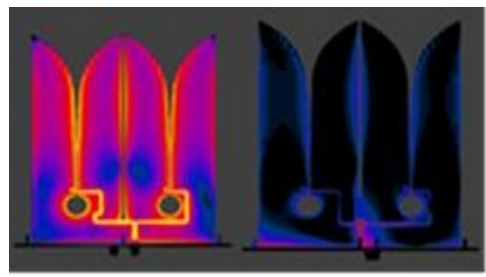

Figure 10-1. Current distribution of the cross polarized planes of the antenna at $0.9 \mathrm{GHz}$.

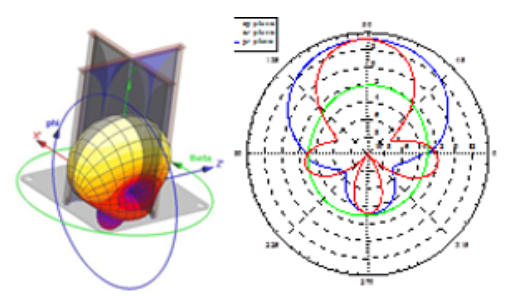

Figure 10-2. Radiation patterns at $0.9 \mathrm{GHz}$.

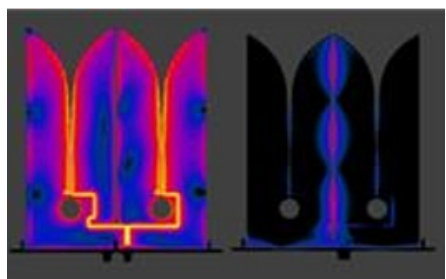


Figure 11-1. Current distribution of the cross polarized planes of the antenna at $1.9 \mathrm{GHz}$.

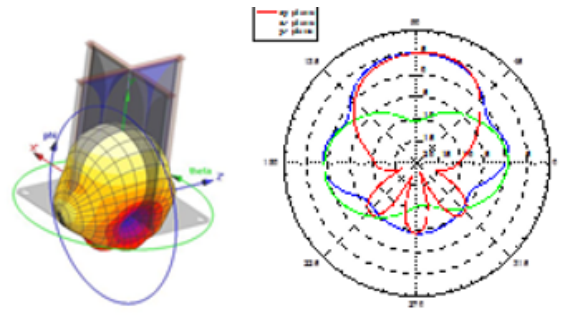

Figure 11-2. Radiation patterns at $1.9 \mathrm{GHz}$.
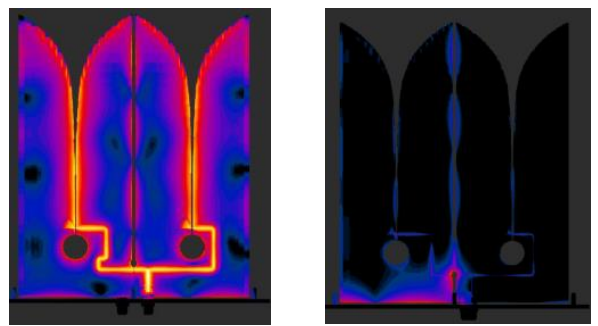

Figure 12-1. Current distribution of the cross polarized planes of the antenna at $3.6 \mathrm{GHz}$.
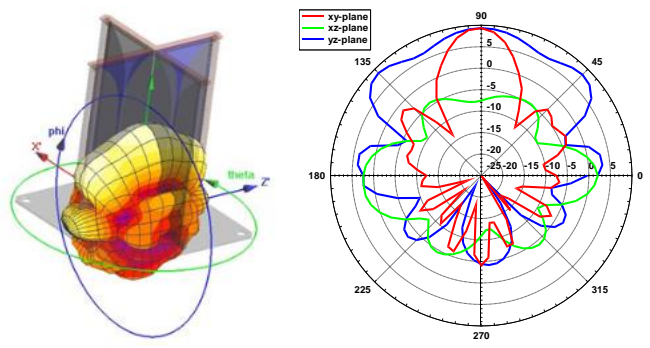

Figure 12-2. Radiation patterns at $3.6 \mathrm{GHz}$.
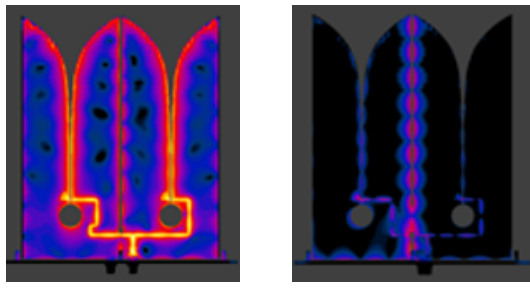

Figure 13-1. Current distribution of the cross polarized planes of the antenna at $5.5 \mathrm{GHz}$.
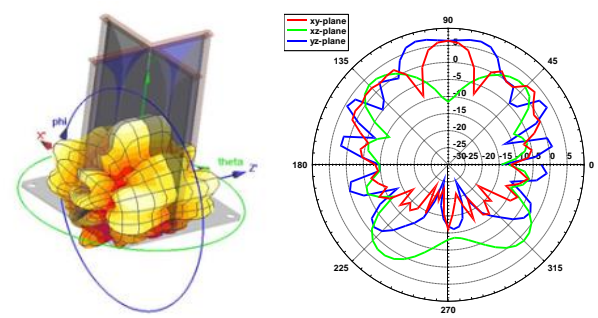

Figure 13-2. Radiation patterns at $5.5 \mathrm{GHz}$.

\section{Conclusion}

In this paper, SPEAG's SEMCAD software was employed to modify and optimize the studied antenna.
Frequency bands used by LTE-A mobile communication were referenced to select four frequency points representing low, medium, and high frequencies to perform simulation calculations. The following relevant antenna performance parameters were determined: the operating VSWR of the antenna (Fig. 7), isolation of dual-polarized antenna, current distribution of the primary and cross polarized planes of the antenna (Figs $10-1,11-1,12-1$, and 13-1), and the two- and threedimensional radiation efficiency patterns of the antenna. The experimental data of this study indicated that the performance of this antenna in the $0.7-6.0 \mathrm{GHz}$ frequency bands satisfy the requirements of a broadband antenna.

Although the power divider circuit and the coupling and feeding circuit structures used in the antenna are simple, this study found that the dimension between the microstrip line and tapered slot line is a key factor. If the dimension between the microstrip feed line and tapered slot line and the diameter of the circular hole are modified, the impedance characteristics of the antenna can be further optimized. This will be studied in the next stage of research. The selection of the thickness of the PCB dielectric slab and its relative dielectric constant also affect the antenna dimensions and performance. The antenna developed in this study did not use a PCB dielectric slab with a higher dielectric constant. If a PCB dielectric slab with higher dielectric constant was used to design this antenna, a lower operating bandwidth could be obtained under the same volume, and the same performance requirements achieved.

This study analyzed the electromagnetic performance of a $2 \times 2$ Vivaldi array broadband antenna. According to the data of the simulation design, its characteristics exhibited excellent results. The $2 \times 2$ broadband antenna array is expected to be applied in a MIMO OTA antenna array anechoic chamber for mobile communication. This study also revealed that this antenna has favorable power divider and coupling and feeding circuit design, which reduces insertion loss and has excellent radiation directivity. Its characteristics of small size, light weight, and minimal cross-sectional area of propagation direction can reduce the multipath reflection and interference in antenna array systems. The author used a cone-shaped electromagnetic wave absorber to perform suitable coating of the antenna and used its coating to reduce the radiation capability of each side and the back, excluding the specified 30-degree half-value angle of the radiation direction. This reduced the multipath reflection and interference in applications in the anechoic chamber. Application of the developed antenna in existing MIMO OTA measurement systems should result in lower multipath reflection produced by lateral radiation, and therefore, it is more suitable for use in the MIMO OTA measurement system to obtain more accurate results. The antenna should perform favorably in applications in the MIMO OTA measurement system of the highly popular mobile communication field.

\section{References}


1. S.G. Kim, K. Chang, Proc. Antennas Propag Soc Int Symp. 2269-2272 (2004).

2. Y.Q. Yang, C.M. Zhang, S. Lin, A.E. Fathy, Proc. Antennas Propaga Soc Int Symp. 606-609 (2005).

3. P.J. Gibson, Proc. 9th Eur Microwav Conf. Brighton, UK. 101-105 (1979).

4. E. Gazit, Proce. IEE Proc Microwav Antennas Propag. 135, 2, 89-92 (1988).

5. L.P.S. Hall, P. Newham, Electron Lett. 29 (2005).

6. I. Linardou, C. Migliaccio, J.M. Laheurte, Electron Lett, 35, 25, 2160-2161 (1999). 